\title{
Demonstration of a microfluidic polarimeter
}

\author{
Rajitha Papukutty Rajan*a, Ambarish Ghosh ${ }^{\sim a}$ \\ a Indian Institute of Science, Bangalore, India 560012
}

\begin{abstract}
Traditional methods of detecting chiral molecules, such as optical rotation are not suitable for miniaturization, since, the magnitude of the rotation of polarization scales down linearly with the optical path length of the device. Since the origin of optical activity is due to difference of refractive indices between the two circularly polarized states of light, it is possible to detect chiral media by measuring the dependence of the angles of refraction on the polarization state of the incident light. This however is a weak effect and hence requires sensitive optical detection schemes, based on novel polarization modulation techniques. The device can be scaled down for applications involving small sample volumes. Fabrication details of a prototype microfluidic device are described.
\end{abstract}

\section{INTRODUCTION}

Chiral molecules are not superimposable on their mirror images and show optical activity ${ }^{1}$. Most biologically relevant molecules are chiral in nature, which show a strong selectivity in their reactions with other chiral molecules. Accordingly, a large percentage of drugs, which can be synthesized as either of the enantiomers; have only one of the forms approved by the FDA. The detection and separation of chiral molecules is therefore of great importance to the pharmaceutical industry. The traditional method of detecting chiral molecules is by optical rotation, which refers to the rotation of the plane of polarization of a linearly polarized light beam as it travels through a chiral medium. This is due to the difference of refractive indices (circular birefringence) between the two circularly polarized components of light in a medium with no mirror symmetry. Since, linearly polarized light beam can be considered to be a combination of left and right circularly polarized light, the two cp components acquire different phases as they travel through the medium, thus causing a rotation in the plane of polarization.

Unfortunately, the measured value of optical rotation scales down linearly with the optical path length and therefore this method is not suited of miniaturization. With recent advancement in microfluidic devices and integrated lab-on-a-chip systems, there is a need of integrating the measurement of optical activity with a simple microscale device. As we show in this paper, this requires novel device fabrication and polarization modulation techniques, combined with a new way of detecting optically active substances.

It was Fresnel who first showed that optically active substance transmits right circularly polarized light with a different velocity from left circularly polarized light which results in a small difference in the angle of refraction when a light beam refracts at the interface of a chiral medium. Since the origin of optical activity is due to difference of refractive indices between the two circularly polarized states of light, it is possible to detect chiral media by measuring the refraction angles of the circularly polarized light beams ${ }^{2-3}$. Since, refraction is an interface phenomenon, large optical path lengths are not necessary, allowing the device to be miniaturized. Furthermore, by placing several such interfaces in particular geometries; it is possible to enhance the difference of the angles of refraction, which increases linearly with the number of interfaces ${ }^{3}$. 
This paper is divided in three parts. In the first part, we estimate the differences between the angles of refraction for a chiral medium. The second part describes results of the measurements from a macroscopic experimental chamber, where the limit of detection of minimum angular deviations is described in great detail. The final part contains the details of fabrication of a microfluidic device which can be used in such measurements.

\section{THEORY}

Consider linearly polarized light from an achiral medium (refractive index, $\mathrm{n}_{0}$ ) incident at an angle $\mathrm{i}$ on a chiral medium characterized by two refractive indices, $n_{+}$and $n_{-}$for the two circularly polarized components. Upon refraction, the linearly polarized light beam is split into two cp components with refracted angles $r_{-}$and $r_{+}$as shown in figure 1 . These refracted lights obey Snell's law separately:

$$
\mathrm{n}_{0} \sin \mathrm{i}=\mathrm{n} \_\sin \mathrm{r}_{-}=\mathrm{n}_{+} \sin \mathrm{r}_{+}
$$

$\mathrm{n}_{0}$ is refractive index of the achiral medium, $\mathrm{i}$ is the angle of incidence, and $\mathrm{n}_{-}$and $\mathrm{n}_{+}$are the refractive index of left and right circularly polarized light respectively. It should be noted that under most circumstances, the differences between the refractive indices in a chiral medium is more than six orders of magnitude lower than the average refractive index, which is why the splitting of the beam at a single interface cannot be seen with naked eye.

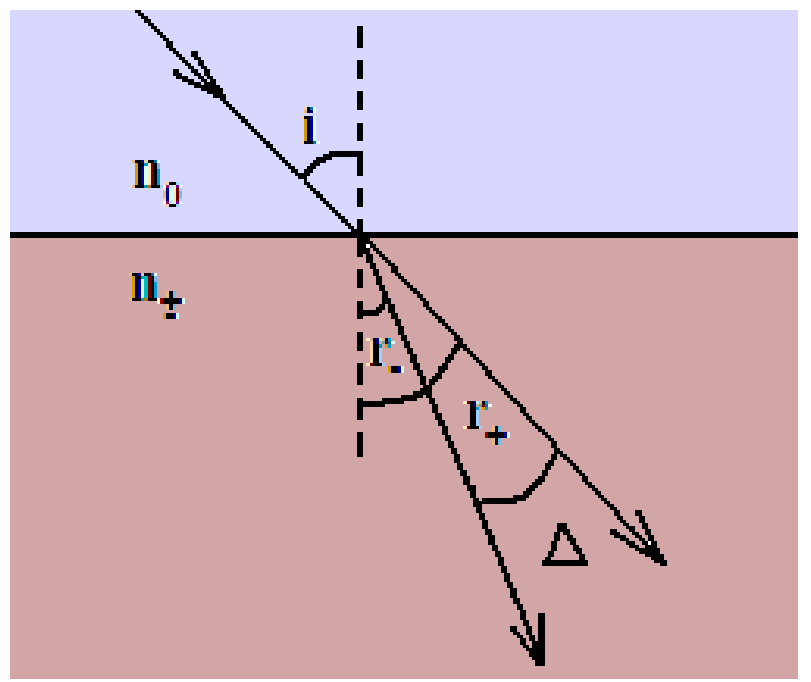

Figure 1. Shows splitting of a linearly polarized light beam when it is incident on a chiral medium from an achiral medium.

The optical rotation of two circularly polarized lights is typically expressed as

$$
\alpha=\pi \mathrm{l}(\Delta \mathrm{n}) / \lambda
$$

where,

$$
\Delta \mathrm{n}=\mathrm{n}_{-}-\mathrm{n}_{+}
$$

where $\lambda$ is the wavelength and 1 is the distance traveled. Thus if the deviation or the difference between the refracted angles of right and left circularly polarized light is $\Delta$, then we can write

$$
\Delta=(\tan \Phi * \Delta \mathrm{n}) / \mathrm{n}
$$


where $\Phi$ is the average of refracted angles at the second interface and $n$ is the average of the two refractive indices in the chiral medium.

It is important to note that by placing another interface at an appropriate angle, the angular deviation can be doubled. By placing multiple interfaces, it is possible to increase the angular deviation many-fold, which is the basis of the microfluidic devices used for chiro-optical deflection measurements.

\section{MEASUREMENT}

The setup to measure the angular deviation between right and left circularly polarized light is shown schematically in Fig. 1. Monochromatic light of wavelength $632.8 \mathrm{~nm}$ from a He-Ne laser $(5 \mathrm{~mW})$ is transmitted through a polarization modulation system, and then through a prism shaped device (not microfluidic) containing a chiral liquid. We have tried various types of polarization modulation schemes, such as Pockel's cells, photoelastic modulators (PEM), liquid crystals etc, and the long term stability was always the best that involved a scheme with two modulators. The position of the beam is measured synchronously with a position sensitive detector and lockin amplifiers. To ensure that the deflection measurements were indeed due to a chiral medium, we measured $\Delta$ as a function of the strength of optical activity, and found a linear dependence. This data was taken with a strongly optically active medium and is not shown. Upon insertion of a half wave plate before the prism, the sign of $\Delta$. was reversed (as expected). Note that the polarization of the cp beams are expected to become somewhat elliptical ${ }^{4}$ at each refracting interface, due to the difference of Fresnel transmission coefficients between the $\mathrm{s}$ and the $\mathrm{p}$ beams, which has not been accounted for in the theoretical estimate shown before.

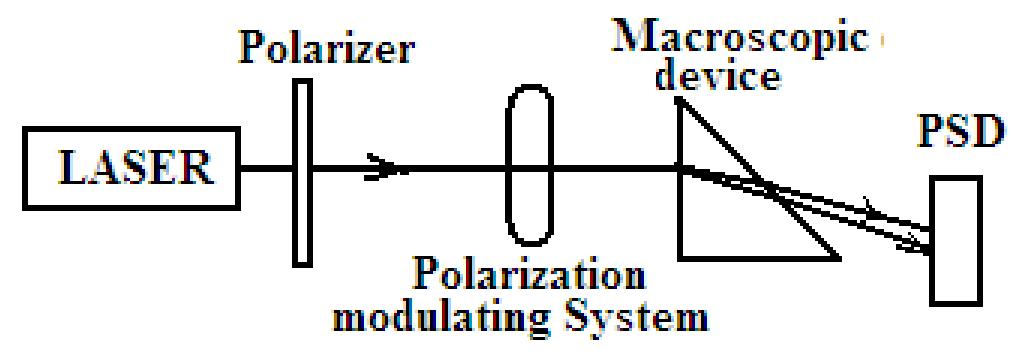

Figure 2. Schematic of the experimental set-up

The macroscopic cell was made with simply holding glass slides together with epoxy, making a prism shaped device that can be filled with different liquids easily. As a model system, we studied glucose in water and measured the angular deviation as a function of concentration of the solution. The dependence of $\Delta$ was measured as function of glucose concentration, and is shown in Fig. 3. It is important to note that typical blood glucose level of a normal human being is around $0.72 \mathrm{mg} / \mathrm{ml}$, which is slightly lower than what we can measure with 10 seconds of data acquisition. The present setup was very sensitive to vibrations, and therefore by moving the setup to a vibration isolated table in future we expect to increase the $\mathrm{S} / \mathrm{N}$ ratio. The current noise floor limits the measurements to concentration of $\sim 10 \mathrm{mg} / \mathrm{ml}$ glucose. Note that we have only measured D-glucose in this report; the sign of the angular deviation is expected to reverse for L-glucose. 


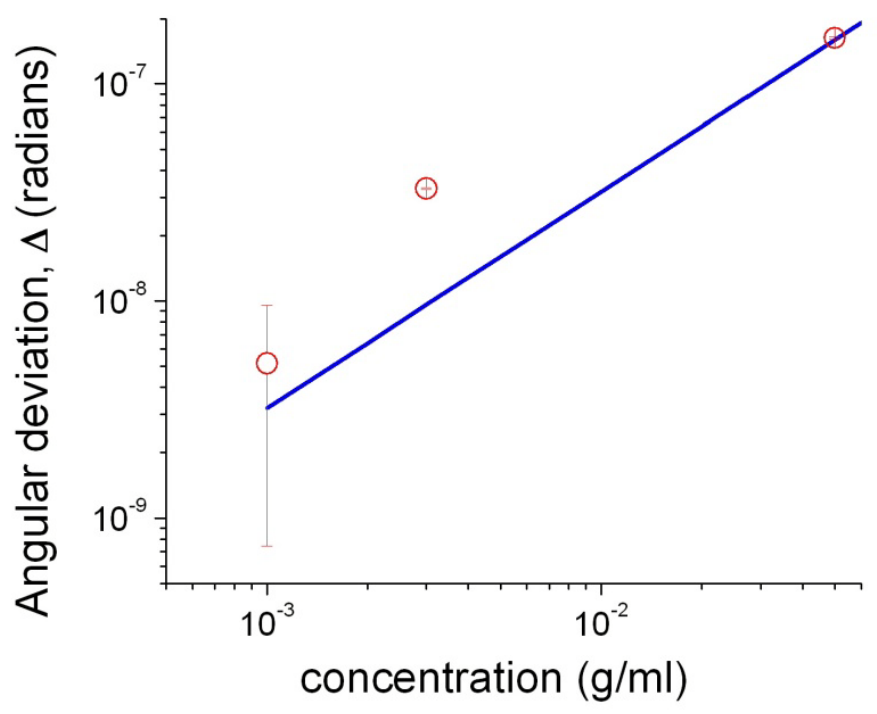

Figure 3: Angular deviation as a function of concentration of D-glucose in water. The blue line corresponds to the expected deviation, while red circles are experimental points averaged over 10 seconds.

One possible way to increase the signal-to-noise ratio of a measurement is to average for a long time. We studied the long term stability of our measurements for a long time (20 minutes) and found excellent long term stability. This is shown in Fig. 4, where the deviation is plotted as a time series for different glucose concentrations: $1 \mathrm{mg} / \mathrm{ml}, 3 \mathrm{mg} / \mathrm{ml}$ and $50 \mathrm{mg} / \mathrm{ml}$ ). Note the lack of a long term drift in the plots. Although not attempted, this implies that by averaging it for 20 minutes, we may be able to improve the signal to noise ratio by about 10 times, implying a limit of detection better than $1 \mathrm{mg} / \mathrm{ml}$. This will be studied in near future.

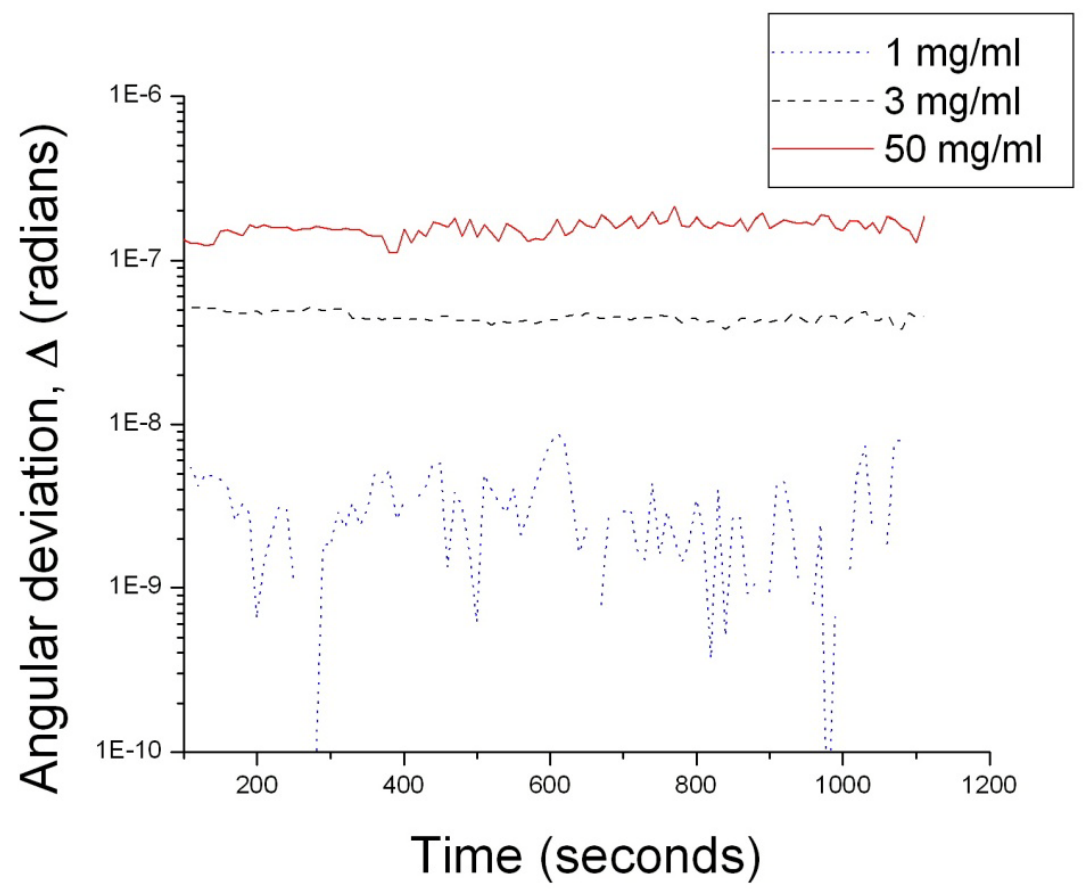

Figure 4: Plot of the measured angular deviation as a time series. The measured values have good long term stability 


\section{DEVICE FABRICATION}

Here, we describe the fabrication of a prism shaped microfluidic device made of Polydimethylsiloxane (PDMS). The advantages of PDMS are the ease of fabrication and optical transparency within the visible spectrum, which ensures its applicability in optical microfluidic systems.

PDMS was prepared by mixing the base and the curing agent in the ratio 10:1 weight. It's then poured into a prism confined within microscopic glass slides to confine the follow of PDMS. The PDMS is baked to make it hard. Once the PDMS is hardened, the prism is removed to get a prism shaped structure in the PDMS. Then the PDMS is peeled and bonded to a glass substrate. Laser light of $632.8 \mathrm{~nm}$ was passed through the mold and it was observed that (1) PDMS had good optical finish, (2) the beam was found to refract as it passed from one medium to another within the mold, (3) the beam quality before entering and after exiting the mold was same. Figure 5 show the refraction of light as it passes through the device (containing air)

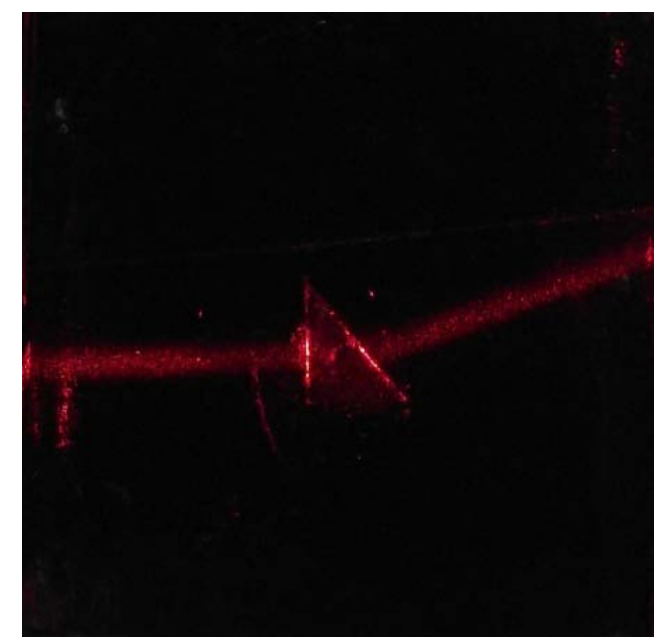

Figure 5. Refraction of laser beam as it passes through the prism made in PDMS.

The angular deviation increases as the number of interfaces is increased. In Fig. 6, we show a device that has multiple prism shaped devices with relevant fluidic inlets and outlets. The method of fabrication is similar to what has been shown before. The typical size of the device shown here is around a few mm. Alternate prisms need to be filled with alternate enantiomeric forms of the chiral molecule, which may result in large beam deflections.

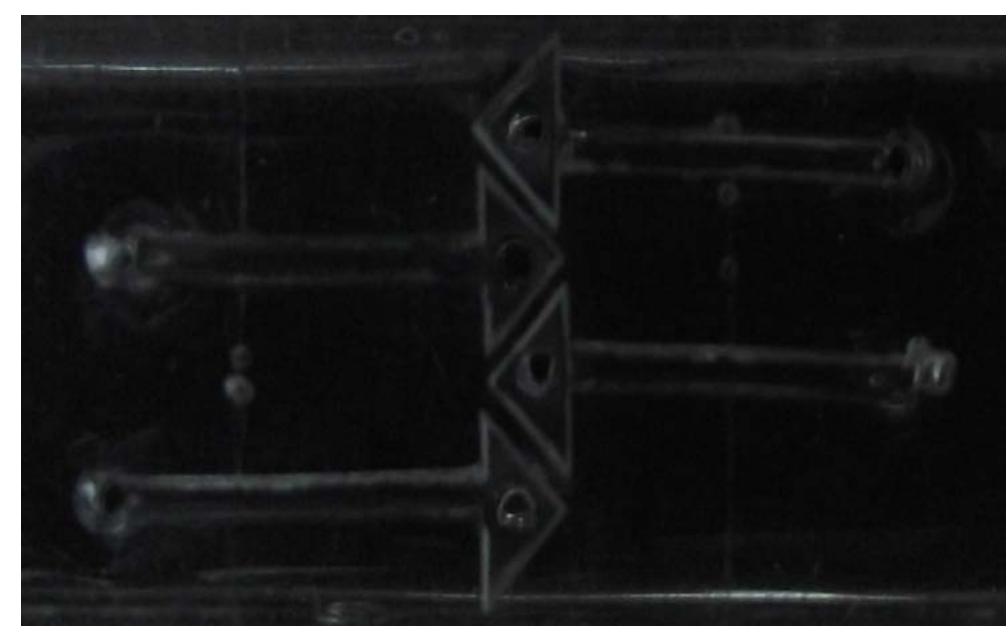

Figure 6. PDMS microfluidic device containing multiple interfaces 


\section{CONCLUSION}

We describe a method and a device to detect microfluidic quantities of a dilute solution of chiral molecules. The method is based on measuring the angular deflections of circularly polarized light beams as it refracts at the interface of a chiral medium. While there is scope of improvement (such as using other measurement schemes ${ }^{5}$ ) in reducing the noise floor of the measurement of the angular deflections, the measurement setup has excellent long term stability, which may help in improving the $\mathrm{S} / \mathrm{N}$ ratio by long time averaging. We also show a microfluidic device based on PDMS which may aid in increasing the angular deviation (by having multiple refracting interfaces), thus improving the limit of detection in chiro-optical measurements. It will be interesting

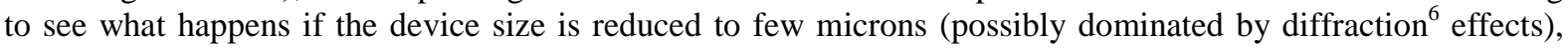
that may be fabricated easily using standard lithographic techniques.

We thank DST Fast Track Scheme, DBT and ADA (NPMASS) for funding this work. Loan of the polarization modulator from Applied Photonics Initiative, IISc is gratefully acknowledged.

\section{REFERENCES}

[1] L. D. Barron,[ Molecular Light Scattering and Optical Activity],Cambridge University Press, Cambridge, England (2004).

[2] Ambarish Ghosh, Winfield Hill, and Peer Fischer, "Observation of the Faraday effect via beam deflection in a longitudinal magnetic field”, Phys.Rev. A, 76, 055402 (2007)

[3] A. Ghosh and P. Fischer, “Chiral Molecules Split Light: Reflection and Refraction in a Chiral Liquid”, Phys. Rev. Lett. 97, 173002 (2006).

[4] M. Silverman, "Reflection and refraction at the surface of a chiral medium: comparison of gyrotropic constitutive relations invariant or noninvariant under a duality transformation”, J. Opt. Soc. Am. A,4(6), 1145-1145 (1987).

[5] Marcel Pfeifer, Peer Fischer, “Weak value amplified optical activity measurements”, Opt. Express, 19(17), 1650816517 (2011)

[6] Ambarish Ghosh, Furqan M. Fazal, and Peer Fischer, "Circular differential double diffraction in chiral media”, 32, 13 (2007). 